\title{
Actively maintained lipid nanodomains in biomembranes
}

\author{
Jordi Gómez, Francesc Sagués, and Ramon Reigada \\ Departament de Química-Física, Universitat de Barcelona, Avda. Diagonal 647, 08028 Barcelona, Spain
}

(Received 6 November 2006; revised manuscript received 6 June 2007; published 11 February 2008)

\begin{abstract}
Lipid rafts, defined as domains rich in cholesterol and sphingolipids, are involved in many important plasma membrane functions. Recent studies suggest that the way cells handle membrane cholesterol is fundamental in the formation of such lateral heterogeneities. We propose to model the plasma membrane as a nonequilibrium phase-separating system where cholesterol is dynamically incorporated and released. The model shows how cellular regulation of membrane cholesterol may determine the nanoscale lipid organization when the lipid mixture is close to a phase separation boundary, providing a plausible mechanism for raft formation in vivo.
\end{abstract}

DOI: 10.1103/PhysRevE.77.021907 PACS number(s): 87.16.D-, 82.39.-k, 82.20.Wt, 82.40.Ck

\section{INTRODUCTION}

During the past decade, a large number of studies revealed that a cell membrane is not the homogeneous and passive bilayer described in the "fluid mosaic model" [1], but a lateral heterogeneous medium with a complex nanoscale lipidic organization [2]. To provide a more accurate description, Simons and Ikonen [3] proposed the concept of rafts to refer to lateral domains rich in saturated lipids and cholesterol, dispersed throughout a phase rich in unsaturated lipids. Such structures are estimated to be in the tens to few hundreds nanometers range $[4,5]$, and are endowed with membrane protein sorting and signal transduction functions [6,7]. Furthermore, they have been recently unveiled as dynamic and scale-dependent structures; namely, their size and stability may dynamically change under specific signals or stimulus, contributing to the diversification of cellular responses [8-10].

Domain formation in multicomponent systems is commonly associated with the existence of dissimilar affinities between the components. Experimental evidence for strong differential interactions of cholesterol with usual membrane lipids is well known in monolayers and bilayers [11], and is generally manifested in massive phase separation in giant vesicles [12,13]. Cholesterol, having a semirigid tail, has a larger affinity for saturated lipids, and this is usually assumed to be the driving force of raft formation in living cell membranes. In phase separating systems, however, coexistent domains continuously grow (minimizing the boundary energy) until reaching, at equilibrium, a length scale of the order of the system size. Thus thermodynamic arguments alone cannot explain either the nanometric size of rafts, nor (and even less) their active behavior. This prompts the use of dynamic concepts to describe the behavior of cell membranes.

Nonequilibrium pattern formation in condensed soft matter [14] has attracted much attention of physicists, specially when approaching the biomembrane context [15]. In contrast to equilibrium structures whose wavelength is dictated only by the interactions between the molecular components [16], the length scale of actively maintained patterns in labile condensed systems results from the competition of thermodynamic forces and kinetic parameters accounting for transport and relaxation processes [14]. We argue that this perspective can be useful to explain the spatial characteristics of rafts in biomembranes.
Cell membranes are continuously subjected to intro- and extracellular fluxes involving energy and mass transport. Among many others, recent experiments [8,9] revealed that raft organization is extremely sensitive to cholesterol homeostasis. Mammalian cells synthesize and transport cholesterol to the plasma membrane, and at the same time, cholesterol is continuously released to the external circulation [17]. In a recent model presented by Foret [18], lipid exchange with the surrounding medium is proposed to lead to the formation of stable finite size domains if simultaneously combined with the lipid phase separation. A similar idea is proposed by Turner et al. [19] for a system with three components (two lipids and cholesterol) by means of an aggregation kinetics scheme. Here, using a combined extension of the two aforementioned proposals, we illustrate how cholesterol recycling may regulate raft size in cell membranes at realistic biological conditions. According to the proposed picture, cholesterol fluxes and raft formation would be intimately connected, setting a possible pathway of the cellular signal-response machinery: A given stimulus may modify cholesterol fluxes, causing an alteration of the membrane raft organization that, in turn, may change the membrane functionality as a cellular response.

This paper is organized as follows. In Sec. II the model approach is described and its kinetic equations are derived. The linear stability analysis of these equations is performed and the main results are presented in Sec. III. Numerical simulations are carried out and some representative results are shown in Sec. IV. We conclude with a brief summary in Sec. V.

\section{MODEL}

Our approach is based on analytical and numerical treatments of a simple nonequilibrium model for a ternary membrane subjected to a continuous recycling of one of the components. Two kinds of generic lipids, saturated $(A)$ and unsaturated $(B)$, fully occupy a 2D triangular lattice, $\phi$ being the molar fraction of saturated lipid. The third component, cholesterol $(C)$, is allowed to reside intercalated in the corresponding honeycomb lattice (see Fig. 1), $c$ being its occupancy fraction. The membrane molar fraction of $A, B$, and $C$ components are $\phi /(1+c),(1-\phi) /(1+c)$, and $c /(1+c)$, respectively. 


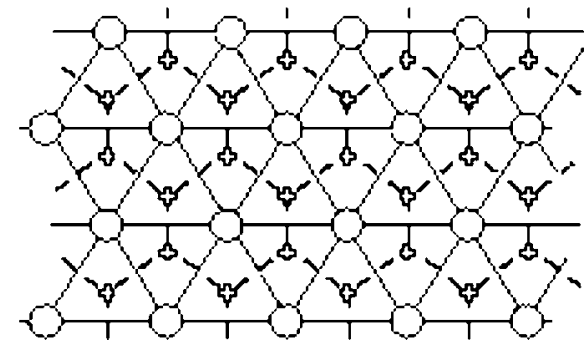

FIG. 1. Lipid lattices used as the starting point of our model. Saturated and unsaturated lipids fully occupy the triangular lattice of circular nodes. Cholesterols are intercalated between the lipids and occupy part of the cross nodes forming the honeycomb lattice.

The free energy of the system in units of $k_{B} T$ reads

$$
\mathcal{F}[\phi, c]=N \int\left(f[\phi, c]+\frac{\gamma}{2}|\nabla \phi|^{2}\right) d s,
$$

where the integration is performed over the membrane area, $N$ is the number of lipids per area, and the square-gradient contribution is the energy cost associated with a nonuniform lipid distribution. Line tension $\gamma$ can be estimated from Cahn-Hilliard theory [20] as $\gamma \approx \frac{1}{2} u l_{0}^{2}$, where $u$ is the typical interaction energy (on the order of $k_{B} T$ ), and $l_{0}$ is the characteristic interfacial width here considered on the order of a few molecular diameters $\left(l_{0} \approx 5 \mathrm{~nm}\right)$. The application of concepts from regular solution theory [21] to the proposed lipid and cholesterol lattices leads to the following local meanfield free energy density,

$$
\begin{aligned}
f[\phi, c]= & \phi \ln (\phi)+(1-\phi) \ln (1-\phi) \\
& +2 c \ln (c)+2(1-c) \ln (1-c) \\
& +Z_{a b} W_{a b} \phi(1-\phi)+Z_{c} W_{c} c(1-2 \phi) .
\end{aligned}
$$

The first four terms are the entropic contributions of both lattices (having the cholesterol lattice twice the number of sites than the lipid lattice), and the fifth and sixth terms account for the lipid-lipid and cholesterol-lipid interactions, respectively. $W_{a b}$ is the difference between the interaction energy of a pair $A B$ and the average of interaction energies of pairs $A A$ and $B B$. The interaction of cholesterol with the lipid mixture is denoted $W_{c}$, accounting for the energy cost of a $B C$ pair, and for simplicity, assumed equal to the energy gain of a pair $A C$. Both interaction parameters are taken to be positive. $Z_{a b}=Z_{c}=6$ are, respectively, the coordination numbers of the lipid and cholesterol lattices for a given lipid molecule. Notice that in Eq. (2), for simplicity, only lipidlipid and cholesterol-lipid interactions are taken into account. Coherently, the absence of cholesterol-cholesterol interactions allows us to neglect a square-gradient term for the cholesterol distribution in Eq. (1). Inclusion of such interactions does not substantially modify the main results presented in this paper.

Temporal evolution of the compositional fields is obtained by applying the constitutive relations from linear nonequilibrium thermodynamics, and mass balance equations to give [22]

$$
\begin{gathered}
\frac{\partial \phi}{\partial t}=D \vec{\nabla} \cdot\left[\phi(1-\phi) \overrightarrow{\nabla \mu_{\phi}}\right], \\
\frac{\partial c}{\partial t}=D \vec{\nabla} \cdot\left[c(1-c) \overrightarrow{\nabla \mu_{c}}\right]+J_{\mathrm{ex}}(c),
\end{gathered}
$$

where $D$ is the molecular lipid and cholesterol diffusivity. The exchange term $J_{\mathrm{ex}}(c)$ describes a generic cholesterol recycling process in the membrane. We consider a homogeneous intake flux of cholesterol from the cytoplasmatic medium with frequency $J_{\mathrm{in}}$, and a release of cholesterol to the external circulation with frequency $J_{\text {out }} c$. This results in $J_{\text {ex }}(c)=-J(c-\bar{c})$, where $\bar{c}=J_{\text {in }} / J_{\text {out }}$ is taken to be the averaged cholesterol fraction in the membrane, and $J=J_{\text {out }}$ is assumed to be the characteristic recycling frequency. Notice that the conservation of the total amount of cholesterol $\bar{c}$ as proposed, is the simplest way to introduce a unique time scale $J^{-1}$ for the recycling process. Such a time scale, rather than a detailed description of all possible cholesterol transport processes through the membrane, is the relevant parameter of the model. The chemical potentials, $\mu_{i}$, are written as the functional derivatives of the free energy in Eq. (1) with respect to the corresponding variables,

$$
\mu_{\phi}=\frac{\delta \mathcal{F}[\phi, c]}{N \delta \phi}, \quad \mu_{c}=\frac{\delta \mathcal{F}[\phi, c]}{2 N \delta c},
$$

leading to the final kinetic equations

$$
\begin{gathered}
\frac{\partial \phi}{\partial t}=D \vec{\nabla} \cdot\left[\overrightarrow{\nabla \phi}+\phi(1-\phi)\left(w_{l} \overrightarrow{\nabla \phi}+w_{c} \overrightarrow{\nabla c}-\gamma \vec{\nabla}^{3} \phi\right)\right], \\
\frac{\partial c}{\partial t}=D \vec{\nabla} \cdot\left[\overrightarrow{\nabla c}+c(1-c) w_{c} \overrightarrow{\nabla \phi}\right]-J(c-\bar{c}),
\end{gathered}
$$

where $w_{l}=-2 Z_{a b} W_{a b}$ and $w_{c}=-2 Z_{c} W_{c}$.

\section{LINEAR STABILITY ANALYSIS}

Some important results can be anticipated from the linear stability analysis of the model equations. The onedimensional linear stability of the stationary homogeneous solution $[\phi(x)=\bar{\phi}, c(x)=\bar{c}]$ is tested by adding small wave perturbations $\delta \phi \exp [\omega(q) t+i q x]$ and $\delta c \exp [\omega(q) t+i q x]$, and linearizing Eqs. (6) and (7). The growth rate $\omega(q)$ is calculated as the largest eigenvalue of the Jacobian resulting from the linearization matrix. Hereafter, $\bar{\phi}$ and $\bar{c}$ refer to the total mean lipid and cholesterol variables, respectively.

In the absence of fluxes the critical condition for equilibrium phase separation is expressed in terms of the parameter A,

$$
A=-2-w_{l} \phi^{\prime}+\sqrt{\left(w_{l} \phi^{\prime}\right)^{2}+4 w_{c}^{2} \phi^{\prime} c^{\prime}},
$$

where we have simplified $\phi^{\prime}=\bar{\phi}(1-\bar{\phi})$ and $c^{\prime}=\bar{c}(1-\bar{c})$. For $A<0$ the stability analysis does not predict any unstable mode $[\omega(q)<0 \forall q]$, resulting in a homogeneous mixture of the three components. When $A>0$, the large wavelength 


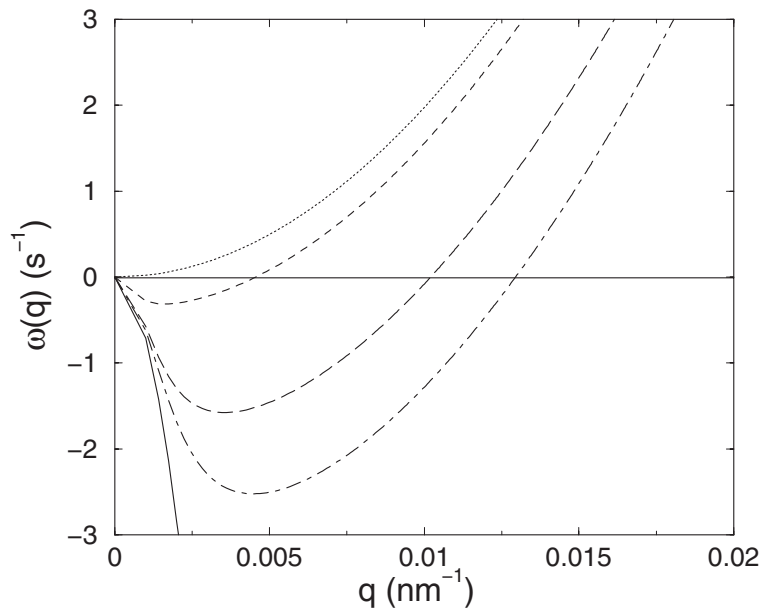

FIG. 2. Growth rates $\omega(q)$ are plotted for the cases $D$ $=1 \mu \mathrm{m}^{2} / \mathrm{s}, W_{a b}=0.1, \gamma=12.5 \mathrm{~nm}^{2}$, and $\bar{\phi}=\bar{c}=0.4$. In the absence of fluxes we compare two different levels of lipid-cholesterol interaction $\left(W_{c}=0\right.$, solid, and $W_{c}=0.3$, dotted). For the case with $W_{c}$ $=0.3$ we compare the effect of different flux rates $\left(J=1 \mathrm{~s}^{-1}\right.$, dashed, $J=5 \mathrm{~s}^{-1}$, long-dashed, and $J=8 \mathrm{~s}^{-1}$, dot-dashed). Fluxes eliminate unstable modes at small wave numbers $q<q_{-}$, selecting a characteristic length $L=\pi / q_{-}$. All curves decay to negative values at large $q$ (not shown).

modes (small $q$ ) become unstable leading to complete phase separation (Fig. 2). However, when fluxes are applied to the latter case, the growth rate $\omega(q)$ is shifted down and the largest wavelength modes are then stabilized, so that segregating domains are predicted to stop growing at a given size determined by the smallest unstable $q=q_{-}$(see Fig. 2). By expanding $\omega(q)$ up to second order on $q^{2}$ one may estimate the smallest unstable mode to be

$$
q_{-}^{2} \approx \frac{2 J}{D A\left(\frac{A+2}{2+2 w_{l} \phi^{\prime}}+1\right)} .
$$

This estimation is exact when the gradient term in Eq. (1) is neglected. Consideration of such a term (as it is done in Fig. 2 ), or even higher order gradient contributions, increases the estimated value for $q_{-}$[23].

The mechanism leading to nonequilibrium pattern size selection is outlined in Eq. (9) as the competition between the short-scale ordering effect of phase separation (denominator) and the large-scale mixing effect of the cholesterol trafficking (numerator). Weak thermodynamic phase separation combined with fast enough cholesterol recycling may lead to stationary domains at the nanometric scale. In Fig. 2 we have chosen a $2: 3$ molar ratio of saturated-unsaturated lipids $(\bar{\phi}$ $=0.4$ ), a $28.5 \%$ of cholesterol in the membrane $(\bar{c}=0.4)$, and a usual diffusivity $D=1 \mu \mathrm{m}^{2} / \mathrm{s}$ [24]. Lipid-lipid differential interactions in membranes are typically small, $W_{a b} \approx 0.1$ at $T=35^{\circ} \mathrm{C}$ [25], insufficient to promote phase separation. However, preferential interaction of cholesterol with saturated lipids, $W_{c} \approx 0.3$ [25], places the system in a two-phase region of the phase diagram but close to the phase separation boundary. In our analysis, this is quantified by a positive but

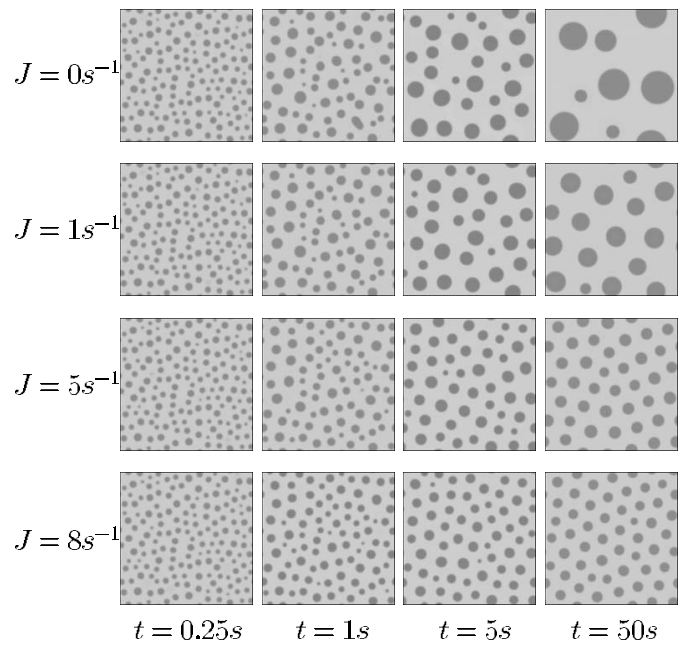

FIG. 3. Temporal evolution of the simulation patterns in a system of $1.25 \mu \mathrm{m} \times 1.25 \mu \mathrm{m}$ obtained with the same parameters as in Fig. 2. Each snapshot corresponds to a grayscale representation of the parameter $\phi$. Darker regions correspond to higher values of the displayed variable. The snapshots for $c$ (not shown) follow the same distribution as those of $\phi$. Only the last temporal snapshots for the nonequilibrium cases $(J \neq 0)$ are practically stationary.

small value for the parameter $A$. This choice is not arbitrary, since membrane lipid mixtures are generally accepted to be very close to a phase separation boundary [8]. In this situation, biological cholesterol exchange frequencies of the order of the $s^{-1}$ [26] lead to structures at realistic nanometric length scales typical of rafts. In Fig. 2 we show how for $J$ $=1,5$, and $8 \mathrm{~s}^{-1}$, the predicted characteristic lengths are about 650, 300, and $240 \mathrm{~nm}$, respectively. From values of the growth rates in Fig. 2 we can also estimate that the time scale for the formation of the stationary nanostructures lies in the range of the fraction of a second.

\section{NUMERICAL RESULTS}

Going beyond linear stability analysis, we numerically solve Eqs. (6) and (7) in a two-dimensional square lattice of $256 \times 256$ sites with periodic boundary conditions. The mesh size is $\Delta x=l_{0}=5 \mathrm{~nm}$, so that each site contains about 50 lipid molecules, validating the coarse-grained nature of the compositional order parameters $\phi$ and $c$. The spatial derivatives are calculated by adopting a simple centered scheme, and a first-order Euler algorithm with time step $\Delta t=2.5 \times 10^{-6} \mathrm{~s}$ is used for the temporal integration. Simulations are started from a homogeneous distribution $[\phi(\vec{r})=\bar{\phi}, c(\vec{r})=\bar{c}]$, slightly perturbed with local variations of $\pm 1 \%$. In Fig. 3 we present the numerical simulations with the same parameter values as in Fig. 2. As a generic behavior, the system is segregated in coarsening circular domains rich in cholesterol and saturated lipids (large $\phi$ and $c$ ). According to the prediction of the linear stability analysis, in the presence of cholesterol recycling the domain growth is halted at a finite and characteristic size that depends on the flux rate. Notice that in our simulations a sort of ordered state in the long-time domain distribution is observed for the nonequilibrium cases. This is 


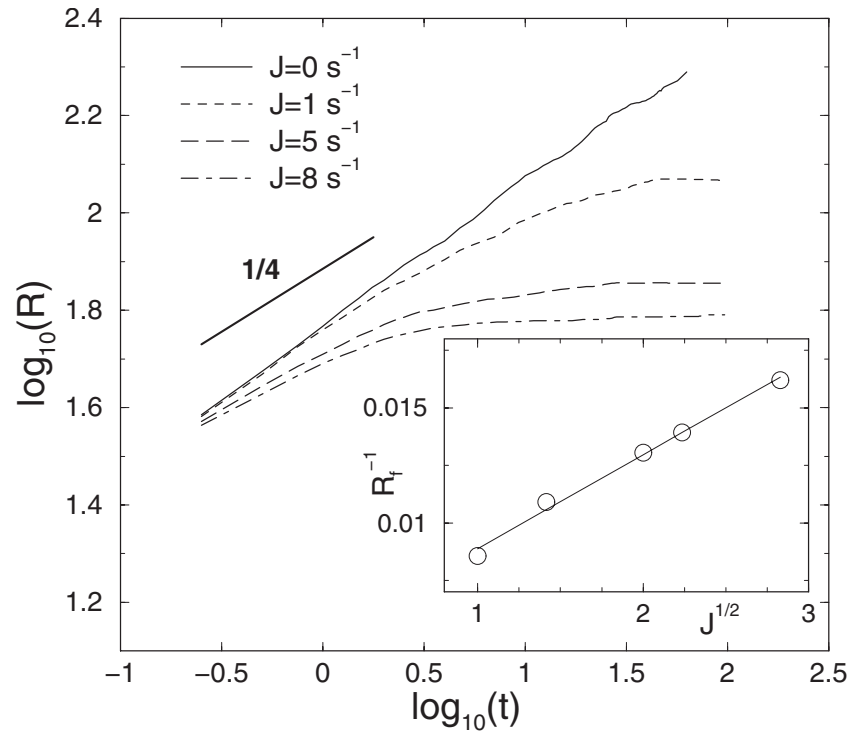

FIG. 4. Log-log temporal evolution of the droplet radius, $R$, for different fluxes and the same parameters as in Figs. 2 and 3. Each curve is computed as the average over simulation patterns obtained from five different initial random distributions. Inset: $R_{f}^{-1}$ vs $J^{1 / 2}$ displays a linear dependence in agreement with Eq. (9).

not surprising at all since ordered spatial arrangements of domains may appear in model systems displaying positive growth rates in a band of wave numbers. The inclusion of noise mimicking thermal and/or cholesterol fluctuations in our equations would result in irregular domain shapes and a disordered spatial domain distribution, without changing the main result presented in this paper.

We quantitatively monitor the growth of the generated domains in Fig. 3 by computing at different times the normalized correlation functions for the $\phi$ variable,

$$
C(r)=\frac{\left\langle\phi\left(\overrightarrow{r^{\prime}}\right) \phi\left(\overrightarrow{r^{\prime}}+\vec{r}\right)\right\rangle-\left\langle\phi\left(\overrightarrow{r^{\prime}}\right)\right\rangle^{2}}{\left\langle\phi\left(\overrightarrow{r^{\prime}}\right)^{2}\right\rangle-\left\langle\phi\left(\overrightarrow{r^{\prime}}\right)\right\rangle^{2}},
$$

where the brackets correspond to a spatial average for all the sites $\overrightarrow{r^{\prime}}$ of the system. The correlation functions for $c$ lead to the same results. A usual estimation for the droplet radius is given by the smallest distance $R$ that satisfies $C(R)=0$. For the equilibrium case we have found the scaling behavior $R(t) \sim t^{\alpha}$ with $\alpha=0.289$ (Fig. 4), close to the asymptotic $1 / 4$ value predicted by the modified Lifshitz-Slyosov theory for concentration dependent diffusivities [27]. In Fig. 4 we also show how the active cholesterol flux stops after a few seconds the coarsening process and leads to stationary domains with an average final radius $R=R_{f}$ of $117 \mathrm{~nm}$ for $J=1 \mathrm{~s}^{-1}$, $72 \mathrm{~nm}$ for $J=5 \mathrm{~s}^{-1}$, and $60 \mathrm{~nm}$ for $J=8 \mathrm{~s}^{-1}$ (Fig. 4). These sizes are smaller than those predicted by the linear stability analysis, as expected, the latter giving an upper limit for the size of the generated structures. The inset in Fig. 4 confirms the dependence of $R_{f}$ (proportional to $q_{-}^{-1}$ ) with the flux rate $J$ predicted in Eq. (9).

Simulation results also evidence how the membrane can dynamically adapt its lateral organization to changes of cho- lesterol trafficking. If the membrane is successively simulated under different flux rates (not shown), the stationary pattern distributions with the corresponding characteristic domain size are obtained after a transient regime that lasts reasonably a few fractions of a second. These transitions present some interesting particularities. When increasing cholesterol traffic rates, our simulations display transient viscous fingering-like distortions of the initially large domains that elongate and later fragment into smaller circular domains. On the other hand, if the recycling frequency is decreased, domains grow following the ripening mechanism, usual in phase separation phenomena. If the fluxes are switched off, a complete equilibrium phase separation in two segregated domains is observed.

\section{CONCLUSIONS AND PERSPECTIVES}

Equilibrium theoretical arguments fail when reproducing the dynamical aspects of raft organization. Here, following the ideas outlined in Refs. [18,19], we have proposed a nonequilibrium description of the cell membrane that captures such a phenomenology at the realistic length and time scales, and agrees with the idea of rafts as actively maintained structures [8,9]. A simpler model for a membrane consisting of two phase separating lipids which are dynamically exchanged has been presented in Ref. [18]. In this model, however, lipid recycling rates leading to nanometric stationary domains are about three orders of magnitude larger than the biological ones. In our model, inclusion of a third component (cholesterol) and the choice of realistic interaction parameters place the lipid mixture close to a phase transition boundary. We demonstrate that it is really in this situation that typical recycling rates (in this case of the cholesterol component) result in nanometric structures. Another model for raft formation based on similar nonequilibrium ingredients has been proposed by Turner et al. [19], whose approach follows a purely temporal aggregation scheme without spatial resolution, quite different from ours but rather complementary.

The model presented here could be extended to retain a higher complexity of cell membranes. For example, local curvature effects coupled to phase separation are known to dramatically slow down the coarsening process [28,29]. The implementation of such an effect in our model would imply that even smaller recycling rates result in nanometric domains. Even more relevant, it is known that not only raft organization affects protein sorting in the plasma membrane, but reversely the presence of proteins affects rafts size and stability (an interesting modeling approach is discussed in Ref. [30]). The effect of membrane proteins is twofold. On the one hand, GPI-anchored proteins locally stabilize cholesterol-saturated lipid assemblies [4], which may act as nucleation centers for raft formation. Such an effect could be incorporated into our model in a very simple way: The lipid mixture could be considered below (instead of above) but close to the phase boundary, and perturbations due to proteins may locally drive the system across it (nucleation). On 
the other hand, protein-protein interactions may result in raft association at a larger scale, as it is seen in experiments with cross-linking antibodies where rafts coalesce and can be even optically visualized $[7,31]$. Further extensions of the presented model should include thus the role of proteins.

\section{ACKNOWLEDGMENTS}

We acknowledge support by SEID through Project No. BQU2003-05042-C02-01 and by DURSI through Project No. 2005SGR00653. We thank Elina Ikonen and Maria García-Parajo for fruitful discussions.
[1] S. J. Singer and G. L. Nicolson, Science 175, 720 (1972).

[2] G. Vereb et al., Proc. Natl. Acad. Sci. U.S.A. 100, 8053 (2003).

[3] K. Simons and E. Ikonen, Nature (London) 387, 569 (1997).

[4] R. G. W. Anderson and K. Jacobson, Science 296, 1821 (2002).

[5] A. Pralle, P. Keller, E.-L. Florin, K. Simons, and J. K. H. Hörber, J. Cell Biol. 148, 997 (2000).

[6] K. Simons and D. Toomre, Mol. Cell. Biol. 1, 31 (2000).

[7] A. D. Douglass and R. D. Vale, Cell 121, 937 (2005).

[8] S. Mayor and M. Rao, Traffic (Oxford, U. K.) 5, 231 (2004).

[9] P. Sharma, R. Varma, R. C. Sarasij, K. Gousset, G. Krishnamoorthy, M. Rao, and S. Mayor, Cell 116, 577 (2004).

[10] W. K. Subczynski and A. Kusumi, Biochim. Biophys. Acta 1610, 231 (2003).

[11] J. R. Silvius, Biochim. Biophys. Acta 1610, 174 (2003).

[12] A. Radhakrishnan and H. McConnell, Proc. Natl. Acad. Sci. U.S.A. 102, 12662 (2005).

[13] S. L. Veatch and S. L. Keller, Phys. Rev. Lett. 94, 148101 (2005).

[14] A. S. Mikhailov and G. Ertl, Science 272, 1596 (1996).

[15] S. Ramaswamy, J. Toner, and J. Prost, Phys. Rev. Lett. 84, 3494 (2000).

[16] M. Seul and D. Andelman, Science 267, 476 (1995).
[17] K. Simons and E. Ikonen, Science 290, 1721 (2000).

[18] L. Foret, Europhys. Lett. 71, 508 (2005).

[19] M. S. Turner, P. Sens, and N. D. Socci, Phys. Rev. Lett. 95, 168301 (2005).

[20] J. W. Cahn and J. E. Hilliard, J. Chem. Phys. 28, 258 (1958).

[21] C. H. P. Lupis, Chemical Thermodynamics of Materials (Elsevier, Amsterdam, 1983).

[22] S. R. deGroot and P. Mazur, Non-equilibrium Thermodynamics (North-Holland, Amsterdam, 1962).

[23] F. Ziebert and W. Zimmermann, Phys. Rev. Lett. 93, 159801 (2004).

[24] M. D. Houslay and K. K. Stanley, Dynamics of Biological Membranes (Wiley, New York, 1982).

[25] P. F. F. Almeida, A. Pokorny, and A. Hinderliter, Biochim. Biophys. Acta 1720, 1 (2005).

[26] T. L. Steck, J. Ye, and Y. Lange, Biophys. J. 83, 2118 (2002).

[27] A. J. Bray and C. L. Emmott, Phys. Rev. B 52, R685 (1995).

[28] P. B. Sunil Kumar, G. Gompper, and R. Lipowsky, Phys. Rev. Lett. 86, 3911 (2001).

[29] T. Taniguchi, Phys. Rev. Lett. 76, 4444 (1996).

[30] K. John and M. Bär, Phys. Rev. Lett. 95, 198101 (2005).

[31] T. Harder, P. Scheiffele, P. Verdake, and K. Simons, J. Cell Biol. 141, 929 (1998). 